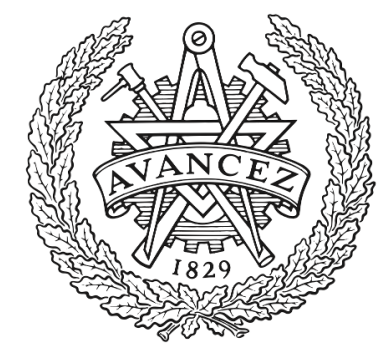

CHALMERS

UNIVERSITY OF TECHNOLOGY

\title{
Pure type systems with judgemental equality
}

Downloaded from: https://research.chalmers.se, 2023-04-26 10:22 UTC

Citation for the original published paper (version of record):

Adams, R. (2006). Pure type systems with judgemental equality. Journal of Functional Programming, 16(2): 219-246. http://dx.doi.org/10.1017/S0956796805005770

N.B. When citing this work, cite the original published paper. 


\title{
Pure type systems with judgemental equality
}

\author{
ROBIN ADAMS* \\ Department of Computer Science, Royal Holloway, University of London, London, UK \\ (e-mail: robin@cs.rhul.ac.uk)
}

\begin{abstract}
In a typing system, there are two approaches that may be taken to the notion of equality. One can use some external relation of convertibility defined on the terms of the grammar, such as $\beta$-convertibility or $\beta \eta$-convertibility; or one can introduce a judgement form for equality into the rules of the typing system itself. For quite some time, it has been an open problem whether the two systems produced by these two choices are equivalent. This problem is essentially the problem of proving that the Subject Reduction property holds in the system with judgemental equality. In this paper, we shall prove that the equivalence holds for all functional Pure Type Systems (PTSs). The proof essentially consists of proving the Church-Rosser Theorem for a typed version of parallel one-step reduction. This method should generalise easily to many typing systems which satisfy the Uniqueness of Types property.
\end{abstract}

\section{Introduction}

When defining a typing system with dependent types, there are two approaches that may be taken to the notion of equality. Firstly, we can use some external syntactic relation defined on the terms of the grammar, such as $\beta$-convertibility or $\beta \eta$-convertibility, as our criterion for equality. This is the method followed by Pure Type Systems (Barendregt, 1992; van Benthem Jutting, 1993), as well as the Calculus of Constructions (Coquand \& Huet, 1988) and the Edinburgh Logical Framework (Harper et al., 1993). In such a system, we typically have a rule of the following form, for replacing a type with a convertible type:

$$
\frac{\Gamma \vdash M: A \quad \Gamma \vdash B \text { type }}{\Gamma \vdash M: B}(A \simeq B)
$$

where $\simeq$ denotes the external convertibility relation, defined without reference to the judgement forms. We shall refer to such systems as type theories with external equality.

The second approach is to introduce separate judgement forms,

$$
\Gamma \vdash M=N: A \text { and } \Gamma \vdash A=B,
$$

for equality of objects and types, and use these judgement forms in the system's rules of deduction. This is the way in which Martin-Löf's Type Theory and Logical

\footnotetext{
* This research began at the University of Manchester supported by EPSRC research grant 00801108, and continued under Project Pythagoras, EPSRC research grant GR/R84108/01.
} 
Framework (Nordström et al., 1990) are normally presented, as well as the recent logical framework $\mathrm{PAL}^{+}$(Luo, 2003), for example. In such a type theory, the rule above is typically replaced with a rule such as

$$
\frac{\Gamma \vdash M: A \quad \Gamma \vdash A=B}{\Gamma \vdash M: B}
$$

making use of the judgement form for equality of types. We shall refer to such systems as type theories with judgemental equality.

For some time, it has been an open question whether the two systems thus produced are equivalent. It would certainly seem to be an important problem, for the presentation with judgemental equality is usually the more convenient to use for theoretical considerations, while almost every implementation of a type system uses an external relation of convertibility to decide equality of terms or types.

The only difficulty in proving the equivalence of the two systems is the establishment of the Subject Reduction property for the system with judgemental equality:

Subject Reduction

If $\Gamma \vdash M: A$ and $M \rightarrow_{\beta} N$, then $\Gamma \vdash M=N: A$.

For such a system, Subject Reduction is essentially the claim that every reduction possible within the external notion of reducibility is reflected by an appropriate equality judgement. Subject Reduction is an important property of type theories with external equality, and establishing that it holds for systems with judgemental equality would also seem to be an important problem.

The problem of the equivalence of the two approaches to equality is mentioned for PTSs with $\beta \eta$-convertibility in Geuvers (1993). The first positive result in this direction was in Coquand (1991), where the equivalence for the system there called Type Theory follows easily as a corollary of the main result. It is also possible, for some systems, to prove the equivalence using the technique of typed operational semantics (Goguen, 1994; Goguen, 1999).

However, both these proof methods rely essentially on the normalisation properties of the type system concerned. This limits the class of systems to which such methods can be applied: normalisability does not hold for every type system, and is notoriously difficult to establish when it does.

In this paper, we shall prove that the equivalence holds for all functional Pure Type Systems (PTSs) (Barendregt, 1992), both normalising and non-normalising; indeed, the proof method is entirely independent of normalisability.

The proof relies essentially on mimicking, in a typed environment, the proof of the Church-Rosser theorem for untyped reduction that uses parallel reduction. We define a relation of typed parallel one-step reduction (TPOSR)

$$
\Theta \models M \triangleright N: A,
$$

and prove that it satisfies the diamond property. For the system with judgemental equality, we find ourselves able to prove subject reduction with respect to this typed reduction, whereas we were unable to do so with untyped reduction. Once we have 
this result, the remainder of the proof is straightforward. Subject Reduction with respect to the untyped reduction follows easily as a corollary.

The method relies heavily on the Uniqueness of Types property, so it does not seem likely it could be extended to an arbitrary PTS; however, it would seem very plausible that it could be adapted to a large number of typing systems that satisfy Uniqueness of Types but cannot be defined as PTSs; for example, the method should easily adapt to cope with the local definitions of $\mathrm{PAL}^{+}$(Luo, 2003), or the inductive types of UTT (Luo, 1994).

\section{Pure type systems}

Given a specification $\mathbf{S}$, we form the PTS $\lambda \mathbf{S}$ as usual, with a single judgement form, $\Gamma \vdash M: A$. We also form a system $\lambda_{=} \mathbf{S}$, a PTS with equality, with two judgement forms, $\Gamma \vdash^{e} M: A$ and $\Gamma \vdash^{e} M=N: A$. We shall give these details fairly briefly; there is a wealth of literature on PTSs, and there is not too much that is innovative in the definition of $\lambda_{=} \mathbf{S}$.

\subsection{Grammar}

A PTS specification $\mathbf{S}$ consists of:

- A set $\mathscr{S}$ of sorts. We shall use $s$ and $t$, possibly adorned with subscripts or accents, as metavariables for sorts.

- A binary relation $\mathscr{A} \subseteq \mathscr{S}^{2}$ of axioms. We write the axiom $(s, t)$ as

$$
s: t
$$

Informally, the axiom $s: t$ indicates that the sort $s$ is an object whose type is the sort $t$.

- A ternary relation $\mathscr{R} \subseteq \mathscr{S}^{3}$ of rules.

Informally, the rule $\left(s_{1}, s_{2}, s_{3}\right)$ indicates that, whenever $A$ is of type $s_{1}$ and, given $x: A, B$ is of type $s_{2}$, then $\Pi x: A . B$ exists and has type $s_{3}$.

We also provide ourselves with an infinite set $\mathscr{V}$ of variables. We shall use $x, y$ and $z$, possibly adorned, as metavariables for variables.

Given a specification $\mathbf{S}$ - in fact, given just the set $\mathscr{S}$ of sorts - the set of terms is defined by the grammar

$$
\operatorname{Term} M::=x|s| \Pi x: M . M|\lambda x: M . M| M M
$$

where $x$ denotes an arbitrary variable and $s$ an arbitrary sort. $x$ is bound within $B$ but not $A$ in the terms $\Pi x: A . B$ and $\lambda x: A . B$. We identify terms up to $\alpha$-convertibility. We shall use capital letters as metavariables for terms.

We define the relations of

$\begin{array}{ll}\text { one-step beta reduction } & \rightarrow_{\beta} \\ \text { beta reduction } & \rightarrow_{\beta} \\ \text { beta conversion } & \simeq_{\beta} \\ \text { parallel one-step reduction } & \rightarrow_{\beta}^{1}\end{array}$


as usual, based on the contraction

$$
(\lambda x: A \cdot M) N \rightsquigarrow_{\beta}[N / x] M
$$

For reference, the inductive definitions we are using for these relations are given in Appendix A.

Theorem 2.1 (Church-Rosser)

If $M \simeq_{\beta} N$, then there exists a term $P$ such that $M \rightarrow_{\beta} P$ and $N \rightarrow_{\beta} P$.

Proof

There are many different proofs of this theorem. In Barendregt (1992), a proof is given that uses the technique of underlining. For a simpler proof using parallel reduction (see Luo, 1994).

A context $\Gamma$ is a sequence of pairs

$$
x_{1}: A_{1}, \ldots, x_{n}: A_{n}
$$

where $x_{1}, \ldots, x_{n}$ are variables, all distinct, and $A_{1}, \ldots, A_{n}$ are terms. Its domain, dom $\Gamma$, is defined to be the set

$$
\left\{x_{1}, \ldots, x_{n}\right\}
$$

We also recall that we say a specification $\mathbf{S}$ is functional iff the following two conditions hold:

- If $s: t, s: t^{\prime} \in \mathscr{A}$, then $t \equiv t^{\prime}$.

- If $\left(s_{1}, s_{2}, s_{3}\right),\left(s_{1}, s_{2}, s_{3}^{\prime}\right) \in \mathscr{R}$, then $s_{3} \equiv s_{3}^{\prime}$.

In Barendregt (1992), it is proven that a PTS with a functional specification has the Uniqueness of Types property.

\subsection{Pure type systems}

Given a specification $\mathbf{S}$, the Pure Type System (PTS) $\lambda \mathbf{S}$ is the system defined as follows.

A judgement in $\lambda \mathbf{S}$ is an expression of the form

$$
\Gamma \vdash M: A
$$

where $\Gamma$ is a context and $M$ and $A$ are terms. The rules of deduction of $\lambda \mathbf{S}$ are given in Figure 1.

The best reference for the basic metatheory of PTSs is still Barendregt (1992), and we shall freely use results from this paper. The only result about PTSs we shall need that is not explicitly given there is Context Conversion:

Lemma 2.2 (Context Conversion)

If $\Gamma, x: A, \Delta \vdash M: B, \Gamma \vdash A^{\prime}: s$, and $A \simeq_{\beta} A^{\prime}$, then

$$
\Gamma, x: A^{\prime}, \Delta \vdash M: B .
$$

This result is an easy corollary of the Weakening and Transitivity lemmas proved in Barendregt (1992). 


\begin{tabular}{|c|c|c|}
\hline (axioms) & $\overline{\vdash s: t}$ & $(s: t \in \mathscr{A})$ \\
\hline \multirow{2}{*}{ (start) } & $\Gamma \vdash A: s$ & \multirow{2}{*}{$(x \notin \operatorname{dom} \Gamma)$} \\
\hline & $\Gamma, x: A \vdash x: A$ & \\
\hline \multirow{2}{*}{ (weak) } & $\Gamma \vdash M: A \quad \Gamma \vdash B: s$ & \multirow{2}{*}{$(x \notin \operatorname{dom} \Gamma)$} \\
\hline & $\Gamma, x: B \vdash M: A$ & \\
\hline \multirow{2}{*}{ (product) } & $\Gamma \vdash A: s_{1} \quad \Gamma, x: A \vdash B: s_{2}$ & \multirow{3}{*}{$\left(\left(s_{1}, s_{2}, s_{3}\right) \in \mathscr{R}\right)$} \\
\hline & $\Gamma \vdash \Pi x: A . B: s_{3}$ & \\
\hline \multirow{2}{*}{ (application) } & $\Gamma \vdash M: \Pi x: A . B \quad \Gamma \vdash N: A$ & \\
\hline & $\Gamma \vdash M N:[N / x] B$ & \multirow{3}{*}{$\left(\left(s_{1}, s_{2}, s_{3}\right) \in \mathscr{R}\right)$} \\
\hline \multirow{2}{*}{ (abstraction) } & $\Gamma, x: A \vdash M: B \quad \Gamma \vdash A: s_{1} \quad \Gamma, x: A \vdash B: s_{2}$ & \\
\hline & 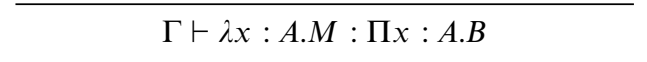 & \\
\hline \multirow{2}{*}{ (conversion) } & $\underline{\Gamma \vdash M: A \quad \Gamma \vdash B: s}$ & \multirow{2}{*}{$\left(A \simeq_{\beta} B\right)$} \\
\hline & $\Gamma \vdash M: B$ & \\
\hline
\end{tabular}

Fig. 1. The rules of deduction of a pure type system.

\subsection{Pure type systems with judgemental equality}

The system $\lambda_{=} \mathbf{S}$, a Pure Type System with Equality, is defined as follows.

A judgement of $\lambda_{=} \mathbf{S}$ is an expression either of the form

$$
\Gamma \vdash^{e} M: A
$$

or

$$
\Gamma \vdash^{e} M=N: A .
$$

The rules of deduction of $\lambda_{=} \mathbf{S}$ are given in Figures 2 and 3. Note that they do not make use of any externally-defined notion of convertibility.

We can develop the metatheory of PTSs with equality quite some way; several lemmas are proven in the Appendix B. However, we find ourselves unable to prove Subject Reduction. See section 3.1 for further discussion on this point.

Our aim in this paper is to prove the following theorem (Theorem 4.6):

\section{Theorem}

If $\mathbf{S}$ is a functional specification, then

1. $\Gamma \vdash^{e} M: A$ iff $\Gamma \vdash M: A$.

2. $\Gamma \vdash^{e} M=N: A$ iff $\Gamma \vdash M: A, \Gamma \vdash N: A$, and $M \simeq_{\beta} N$.

One direction - left-to-right - is easy in each case, and can be proven for an arbitrary PTS: 


\begin{tabular}{|c|c|c|}
\hline \multirow{2}{*}{$(\mathrm{ax})$} & & \multirow{2}{*}{$(s: t \in \mathscr{A})$} \\
\hline & $\vdash^{e} s: t$ & \\
\hline \multirow{2}{*}{ (var) } & $\Gamma \vdash^{e} A: s$ & \multirow{2}{*}{$(x \notin \operatorname{dom} \Gamma)$} \\
\hline & $\overline{\Gamma, x: A \vdash^{e} x: A}$ & \\
\hline \multirow{2}{*}{ (weak) } & $\Gamma \vdash^{e} M: A \quad \Gamma \vdash^{e} B: s$ & \multirow{2}{*}{$(x \notin \operatorname{dom} \Gamma)$} \\
\hline & $\Gamma, x: B \vdash^{e} M: A$ & \\
\hline \multirow{2}{*}{ (weak-eq) } & $\Gamma \vdash^{e} M=N: A \quad \Gamma \vdash^{e} B: s$ & \multirow{2}{*}{$(x \notin \operatorname{dom} \Gamma)$} \\
\hline & $\Gamma, x: B \vdash^{e} M=N: A$ & \\
\hline \multirow[t]{2}{*}{ (prod) } & $\underline{\Gamma \vdash^{e} A: s_{1} \quad \Gamma, x: A \vdash^{e} B: s_{2}}$ & \multirow{2}{*}{$\left(\left(s_{1}, s_{2}, s_{3} \in \mathscr{R}\right)\right.$} \\
\hline & $\Gamma \vdash^{e} \Pi x: A . B: s_{3}$ & \\
\hline \multirow[t]{2}{*}{ (prod-eq) } & $\Gamma \vdash^{e} A=A^{\prime}: s_{1} \quad \Gamma, x: A \vdash^{e} B=B^{\prime}: s_{2}$ & \multirow{2}{*}{$\left(\left(s_{1}, s_{2}, s_{3}\right) \in \mathscr{R}\right)$} \\
\hline & $\Gamma \vdash^{e} \Pi x: A \cdot B=\Pi x: A^{\prime} \cdot B^{\prime}: s_{3}$ & \\
\hline \multirow[t]{2}{*}{ (lambda) } & $\begin{array}{c}\Gamma, x: A \vdash^{e} M: B \quad \Gamma \vdash^{e} A: s_{1} \\
\Gamma, x: A \vdash^{e} B: s_{2} \\
\end{array}$ & \multirow[t]{2}{*}{$\left(\left(s_{1}, s_{2}, s_{3}\right) \in \mathscr{R}\right)$} \\
\hline & $\Gamma \vdash^{e} \lambda x: A . M: \Pi x: A . B$ & \\
\hline \multirow[t]{2}{*}{ (lambda-eq) } & $\begin{array}{c}\Gamma, x: A \vdash^{e} M=M^{\prime}: B \quad \Gamma \vdash^{e} A=A^{\prime}: s_{1} \\
\Gamma, x: A \vdash^{e} B: s_{2}\end{array}$ & \multirow[t]{6}{*}{$\left(\left(s_{1}, s_{2}, s_{3}\right) \in \mathscr{R}\right)$} \\
\hline & $\Gamma \vdash^{e} \lambda x: A . M=\lambda x: A^{\prime} . M^{\prime}: \Pi x: A . B$ & \\
\hline \multirow{2}{*}{$(\mathrm{app})$} & $\Gamma \vdash^{e} M: \Pi x: A . B \quad \Gamma \vdash^{e} N: A$ & \\
\hline & $\Gamma \vdash^{e} M N:[N / x] B$ & \\
\hline \multirow[t]{2}{*}{ (app-eq) } & $\Gamma \vdash^{e} M=M^{\prime}: \Pi x: A . B \quad \Gamma \vdash^{e} N=N^{\prime}: A$ & \\
\hline & $\Gamma \vdash^{e} M N=M^{\prime} N^{\prime}:[N / x] B$ & \\
\hline
\end{tabular}

Fig. 2. The rules of deduction of a pure type system with equality.

\section{Theorem 2.3}

1. If $\Gamma \vdash^{e} M: A$, then $\Gamma \vdash M: A$.

2. If $\Gamma \vdash^{e} M=N: A$, then $\Gamma \vdash M: A, \Gamma \vdash N: A$, and $M \simeq_{\beta} N$.

The proof is by induction on the derivation of the premise in $\lambda_{=} \mathbf{S}$. No case is very difficult, if we have the results of Barendregt (1992) to hand.

The right-to-left direction would be just as simple if only we could use Subject Reduction for the system with judgemental equality. Without it, the proof is very difficult; the remainder of this paper is devoted to this proof (Theorem 4.5). Subject Reduction shall follow as a corollary of this work (Corollary 5.3). 


$$
\begin{array}{cc}
\text { (ref) } & \frac{\Gamma \vdash^{e} M: A}{\Gamma \vdash^{e} M=M: A} \\
\text { (sym) } & \frac{\Gamma \vdash^{e} M=N: A}{\Gamma \vdash^{e} N=M: A} \\
\text { (trans) } & \frac{\Gamma \vdash^{e} M=N: A \quad \Gamma \vdash^{e} N=P: A}{\Gamma \vdash^{e} M=P: A} \\
\text { (conv) } & \frac{\Gamma \vdash^{e} M: A \quad \Gamma \vdash^{e} A=B: s}{\Gamma \vdash^{e} M: B} \\
\text { (conv-eq) } & \frac{\Gamma \vdash^{e} M=N: A \quad \Gamma \vdash^{e} A=B: s}{\Gamma \vdash^{e} M=N: B} \\
& \frac{\Gamma, x: A \vdash^{e} M: B \quad \Gamma \vdash^{e} N: A}{\Gamma \vdash^{e} A: s_{1} \quad \Gamma, x: A \vdash^{e} B: s_{2}} \\
\text { (beta) } \quad\left(\left(s_{1}, s_{2}, s_{3}\right) \in \mathscr{R}\right) \\
\end{array}
$$

Fig. 3. The rules of deduction of a pure type system with equality.

\section{Typed parallel one-step reduction}

\subsection{Digression: The naïve solution}

To provide extra motivation for the various definitions we are about to introduce, and to show from where the idea for our proof method came, let us examine what goes wrong in a naïve attempt to solve the problem we have outlined. We attempt to prove

$$
\text { If } \Gamma \vdash M: A \text { then } \Gamma \vdash^{e} M: A
$$

by induction on the derivation of $\Gamma \vdash M: A$. The only case that gives any difficulty is the rule

$$
\text { (conversion) } \frac{\Gamma \vdash M: A \quad \Gamma \vdash B: s}{\Gamma \vdash M: B}\left(A \simeq_{\beta} B\right)
$$

By the induction hypothesis, $\Gamma \vdash^{e} M: A$ and $\Gamma \vdash^{e} B: s$. We can also show that $\Gamma \vdash^{e} A: t$ for some sort $t$.

By Church-Rosser, there is a term $C$ such that

$$
A \rightarrow{ }_{\beta} C, \quad B \rightarrow_{\beta} C
$$

If only we could prove Subject Reduction for $\lambda_{=} \mathbf{S}$ :

\section{Conjecture 1 (Subject Reduction)}

If $\Gamma \vdash^{e} M: A$ and $M \rightarrow_{\beta} N$, then $\Gamma \vdash^{e} M=N: A$.

Then we could conclude $\Gamma \vdash^{e} A=C: t$ and $\Gamma \vdash^{e} B=C: s$, and the result $\Gamma \vdash^{e} M: B$ would follow by two applications of (conv). Unfortunately, Subject Reduction is very difficult to prove for $\lambda_{=} \mathbf{S}$. 
What goes wrong when we try to prove Subject Reduction by the same method that we use for ordinary PTSs? That is, we attempt to prove simultaneously

1. If $\Gamma \vdash^{e} M: A$ and $\Gamma \rightarrow_{\beta} \Delta$, then $\Delta \vdash^{e} M: A$.

2. If $\Gamma \vdash^{e} M: A$ and $M \rightarrow_{\beta} N$, then $\Gamma \vdash^{e} M=N: A$.

The difficult case, as is to be expected, is where the premise is derived by

$$
\text { (app) } \frac{\Gamma \vdash^{e} \lambda x: A . M: \Pi x: B . C \quad \Gamma \vdash^{e} N: B}{\Gamma \vdash^{e}(\lambda x: A . M) N:[N / x] C}
$$

and the reduction involved is $(\lambda x: A \cdot M) N \rightarrow_{\beta}[N / x] M$.

Inverting the first premise, there must be a rule $\left(s_{1}, s_{2}, s_{3}\right) \in \mathscr{R}$ such that the premise was derived from

$$
\begin{array}{rll}
\Gamma, x: A & \vdash^{e} & M: D \\
\Gamma & \vdash^{e} & A: s_{1} \\
\Gamma, x: A & \vdash^{e} & B: s_{2}
\end{array}
$$

followed by several judgements of the form:

$$
\Gamma \vdash^{e} \Pi x: A . D=X_{1}: t_{1}, \Gamma \vdash^{e} X_{1}=X_{2}: t_{2}, \ldots, \Gamma \vdash^{e} X_{n}=\Pi x: B . C: t_{n+1}
$$

We wish to conclude

$$
\Gamma \vdash^{e}(\lambda x: A \cdot M) N=[N / x] M:[N / x] C .
$$

The obvious way to do so is to use the (beta) rule. To do so, we can try to derive the premise

$$
\Gamma \vdash^{e} N: A
$$

to yield $\Gamma \vdash^{e}(\lambda x: A \cdot M) N=[N / x] M:[N / x] D$, then try to prove $\Gamma \vdash^{e}[N / x] C=$ $[N / x] D: t$ for some $t$. Or we can try to derive

$$
\Gamma, x: B \vdash^{e} M: C,
$$

from which the desired conclusion would follow immediately.

In either case, we would seem to need a lemma of this form:

Conjecture 2 (Injectivity of $\Pi$ )

If $\Gamma \vdash^{e} \Pi x: A . D=\Pi x: B . C: s_{3}$, then there are sorts $s_{1}, s_{2}$ such that $\left(s_{1}, s_{2}, s_{3}\right) \in \mathscr{R}$, and

$$
\Gamma \vdash^{e} A=B: s_{1}, \quad \Gamma, x: A \vdash^{e} D=C: s_{2}
$$

There is no obvious way to prove this statement, as the premise may have been derived via the (trans) rule from a series of judgements of the form

$$
\Gamma \vdash^{e} \Pi x: A . C=X_{1}: Y_{1}, \Gamma \vdash^{e} X_{1}=X_{2}: Y_{2}, \cdots, \Gamma \vdash^{e} X_{n}=\Pi x: B . D: Y_{n+1}
$$

and the terms $X_{1}, X_{2}, \ldots, X_{n}$ may not be $\Pi$-terms. Of course, they must reduce to $\Pi$-terms; but, as we have not yet proven Subject Reduction, this fact is of little use to us. 
We meet a similar problem when proving Subject Reduction for traditional PTSs; we must conclude, from

$$
\Pi x: A . D \simeq_{\beta} \Pi x: B . C,
$$

that

$$
A \simeq_{\beta} B, \quad C \simeq_{\beta} D .
$$

This is quite easily done, using the Church-Rosser Theorem.

Can we do something similar for PTSs with equality? The Church-Rosser Theorem can be proven using parallel one-step reduction. Can we find a relation that plays the same role for PTSs with equality? We need a relation $\Gamma \models M \triangleright N: A$ that:

- satisfies the diamond property: if $\Gamma \models M \triangleright N: A$ and $\Gamma \models M \triangleright P: A$, then there is a term $Q$ such that $\Gamma=N \triangleright Q: A$ and $\Gamma \mid=P \triangleright Q: A$;

- generates the judgemental equality: $\Gamma \vdash^{e} M=N: A$ is the symmetric, transitive closure of $\Gamma \models M \triangleright N: A$;

- respects the formation of $\Pi$-types: if $\Gamma=\Pi x: A . B \triangleright C: s_{3}$, then there are terms $A^{\prime}, B^{\prime}$ and sorts $s_{1}, s_{2}$ such that $\left(s_{1}, s_{2}, s_{3}\right) \in \mathscr{R}, \Gamma \models A \triangleright A^{\prime}: s_{1}$, $\Gamma, x: A=B \triangleright B^{\prime}: s_{2}$, and $C \equiv \Pi x: A^{\prime} . B^{\prime}$.

- satisfies Uniqueness of Types: if $\Gamma \models M \triangleright N: A$ and $\Gamma \models M \triangleright P: B$, then either $A$ and $B$ are the same sort, or $\Gamma \models A \simeq B: s$ for some sort $s$.

(Here, $\Gamma \models A \simeq B: s$ is the equivalence relation generated by $\Gamma \models A \triangleright B: s$.) If so, then we could prove Conjecture 2 above.

It so happens we can give such a relation for a set of labelled terms, in which each application is tagged with the codomain of the applied function. That is, the term constructor for application is of the form

$$
\operatorname{app}_{(x) B}(M N)
$$

for $M$ a term of type $\Pi x: A . B$ and $N$ a term of type $A$.

We shall call the relation $\Theta \models M \triangleright N:$ A typed parallel one-step reduction, or TPOSR, thanks to the analogy between its definition and the definition of parallel one-step reduction in the untyped case. As long as we have the Uniqueness of Types property, the obvious translation from labelled to unlabelled terms

$$
M \mapsto|M|
$$

is a bijection (up to $\beta$-convertibility) between the typable unlabelled terms and the labelled terms typable by TPOSR.

We can define the relation of typed parallel one-step reduction for such a set of terms; the definition is in Figure 4. We could now go on to complete the proof via the method sketched out above. However, now that we have the relation of typed parallel one-step reduction and its Church-Rosser property, a more direct method is open to us. We establish the following lemmas:

Completeness of TPOSR for PTSs If $\Gamma \vdash M: A$, then there exist $\Gamma^{+}, M^{+}, A^{+}$such that $\left|\Gamma^{+}\right| \equiv \Gamma,\left|M^{+}\right| \equiv M,\left|A^{+}\right| \equiv A$, and $\Gamma^{+}=M^{+} \triangleright M^{+}: A^{+}$. (Theorem 4.4) 
Subject Reduction If $\Theta \models M \triangleright X: A$ and $M \rightarrow{ }_{\beta}^{1} N$, then $\Theta \models M \triangleright N: A$. (Theorem 3.10)

Soundness of TPOSR for PTSs with Equality If $\Theta \models M \triangleright N: A$ then $|\Theta| \vdash^{e}|M|=$ $|N|:|A|$. (Theorem 3.5)

Given these three lemmas, the proof of the correspondence between PTSs and PTSs with equality is quite simple (Theorem 4.5).

The Need for Labelled Terms It is perhaps worth noting where exactly the proof breaks down if we try to use unlabelled terms rather than labelled terms.

Let us denote by $\Theta \models A \simeq B: s$ the symmetric, transitive closure of the relation $\Theta \models A \triangleright B$ : s. We shall need the following three results about TPOSR:

Uniqueness of Types (UT)

If $\Theta \models M \triangleright N: A$ and $\Theta \models M \triangleright P: B$, then either $A \equiv B$, or there is a sort $s$ such that $\Theta \models A \simeq B: s$.

Church-Rosser (CR)

If $\Theta \models M \triangleright N: A$ and $\Theta \models M \triangleright P: B$, then there exists $Q$ such that

$$
\begin{array}{lll}
\Theta \models N \triangleright Q: A & \Theta \models P \triangleright Q: A \\
\Theta \models N \triangleright Q: B & \Theta \models P \triangleright Q: B
\end{array}
$$

Injectivity of $\Pi(\operatorname{Inj}(\Pi))$

If $\Theta=\Pi x: A . B \simeq \Pi x: C . D: s_{3}$, then there are sorts $s_{1}, s_{2}$ such that $\left(s_{1}, s_{2}, s_{3}\right) \in \mathscr{R}$ and

$$
\begin{aligned}
\Theta & =A \simeq C: s_{1} \\
\Theta, x: A & =B \simeq D: s_{2}
\end{aligned}
$$

With unlabelled terms, we are able to prove the circle of implications:

$$
\mathrm{UT} \Rightarrow \mathrm{CR} \Rightarrow \operatorname{Inj}(\Pi) \Rightarrow \mathrm{UT}
$$

but we find ourselves unable to prove any one of these individually. In particular, we require Injectivity of $\Pi$ to prove Uniqueness of Types in the case where $M$ is an application.

With labelled terms, we have a way in: we are able to prove Uniqueness of Types. In particular, in the case in which $M$ is a (labelled) application, $\operatorname{app}_{(x) D}(Q R)$, say, then $A$ and $B$ must each be convertible with $[R / x] D$.

The idea of adding typing information to applications has been used several times before; Streicher (1991), for example, uses typed applications to give semantics to the Calculus of Constructions, and examines in detail the relationship between such a system and a system with untyped application.

We have chosen to label applications only with the codomain, not the domain: we do not give applications the form

$$
\operatorname{app}_{\Pi x: A . B}(M N) .
$$

If we did, then the reduction rule for labelled terms would not be left-linear, in the terminology of Combinatory Reduction Systems. We would need to decide when we 
could contract a redex of the form

$$
\operatorname{app}_{\Pi x: A . B}((\lambda x: C \cdot M) N) .
$$

Do we insist that $A$ and $C$ be identical terms, convertible terms, or do we impose no restriction? Each of these choices leads to some added complication.

Having finished describing our plan of attack, we proceed with the details of the proof.

\subsection{Typed parallel one-step reduction}

\section{Definition 3.1 (Labelled Terms)}

Define the set of labelled terms $\mathscr{T}^{+}$by the grammar

$$
\text { Labelled term } M^{+}::=x|s| \lambda x: M^{+} . M^{+}\left|\Pi x: M^{+} \cdot M^{+}\right| \operatorname{app}_{(x) M^{+}}\left(M^{+} M^{+}\right)
$$

We shall refer to the terms we have been dealing with heretofore as unlabelled terms when we wish to distinguish them from labelled terms.

We shall use all the capital letters as metavariables for labelled terms, as well as for unlabelled terms. This should hopefully not cause any confusion; it should be clear from which kind of term is intended in each case.

$x$ is bound within $B$ but not $A$ in $\lambda x: A . B$ and $\Pi x: A . B$. It is bound within $C$ but not $A$ or $B$ within $\operatorname{app}_{(x) C}(A B)$. We identify labelled terms up to $\alpha$-convertibility.

We define a translation

$$
M \mapsto|M|
$$

that maps labelled terms to unlabelled terms thus:

$$
\begin{aligned}
|x| & \equiv x \\
|s| & \equiv s \\
|\lambda x: A \cdot M| & \equiv \lambda x:|A| \cdot|M| \\
|\Pi x: A . B| & \equiv \Pi x:|A| \cdot|B| \\
\left|\operatorname{app}_{(x) A}(M N)\right| & \equiv|M||N|
\end{aligned}
$$

We define a labelled context $\Theta$ to be a sequence of pairs $x_{1}: A_{1}, \ldots, x_{n}: A_{n}$, where $x_{1}, \ldots, x_{n}$ are distinct variables, and $A_{1}, \ldots, A_{n}$ are labelled terms. We shall use $\Theta$ and $\Phi$ as metavariables for labelled contexts.

We extend the translation above to contexts in the obvious manner:

$$
\left|x_{1}: A_{1}, \ldots, x_{n}: A_{n}\right| \equiv x_{1}:\left|A_{1}\right|, \ldots, x_{n}:\left|A_{n}\right|
$$

We define $\beta$-reduction, conversion, etc. on labelled terms as we did for unlabelled terms, based on the contraction

$$
\operatorname{app}_{(x) B}((\lambda x: A \cdot M) N) \rightsquigarrow_{\beta}[N / x] M
$$

Again, the definitions are written out in full in Appendix A for reference.

The following lemma is easily proven: 


\section{Lemma 3.2}

For labelled terms $M, N$, if $M \rightarrow_{\beta} N$ then $|M| \rightarrow_{\beta}|N|$.

It is not that difficult to prove the following converse, either:

\section{Lemma 3.3}

Let $M^{+}$be a labelled term, and $N$ an unlabelled term. If $\left|M^{+}\right| \rightarrow_{\beta} N$, then there exists $N^{+}$such that $\left|N^{+}\right| \equiv N$ and $M^{+} \rightarrow_{\beta} N^{+}$.

Similar results hold for the relations $\rightarrow_{\beta}^{1}$ and $\rightarrow \beta$.

We now define the formal system Typed Parallel One-Step Reduction (TPOSR), whose judgement forms are

$$
\Theta \text { valid and } \Theta \models M \triangleright N: A \text {, }
$$

where $\Theta$ is a labelled context and $M, N, A$ labelled terms. The rules of deduction of TPOSR are given in Figure 4. Note, in particular, the (red) and (exp) rules: we make the definition of typed parallel one-step reduction self-contained, by using the relation as its own criterion for convertibility of types.

We shall use $\Theta=J$ to indicate either of the judgement forms $\Theta$ valid and $\Theta \models M \triangleright N: A$.

We write

$$
\Theta=M \triangleright ?: A
$$

for $\exists N \Theta \models M \triangleright N: A$.

We define $\Theta \models X \triangleright^{+} Y: Z$ to be the transitive closure of $\Theta \models X \triangleright Y: Z$; that is, the relation $\Theta \models X \triangleright^{+} Y: Z$ is defined inductively by

$$
\frac{\Theta \models X \triangleright Y: Z}{\Theta \models X \triangleright^{+} Y: Z} \quad \frac{\Theta \models W \triangleright^{+} X: Z \quad \Theta \models X \triangleright^{+} Y: Z}{\Theta \models W \triangleright^{+} Y: Z}
$$

We also define $\Theta \models X \simeq Y: s$ to be the symmetric, transitive closure of $\Theta \models$ $X \triangleright Y: s$; that is, the relation $\Theta \models X \simeq Y: s$ is defined inductively by

$$
\begin{array}{lll}
\Theta \models X \triangleright Y: s & \Theta \models X \simeq Y: s
\end{array} \quad \frac{\Theta \models X \simeq Y: s \quad \Theta \models Y \simeq Z: s}{\Theta \models X \simeq Y: s} \quad \frac{\Theta \models Y \simeq X: s}{\Theta \models X \simeq Z: s}
$$

Of course, the (red) and (exp) rules give us:

\section{Lemma 3.4}

If $\Theta \models M \triangleright N: A$ and $\Theta \models A \simeq B: s$ then $\Theta \models M \triangleright N: B$.

We can immediately establish that the translation $X \mapsto|X|$ is a sound translation from typed parallel one-step reduction to PTSs with equality:

Theorem 3.5

If $\Theta \models M \triangleright N: A$ then $|\Theta| \vdash^{e}|M|=|N|:|A|$. 


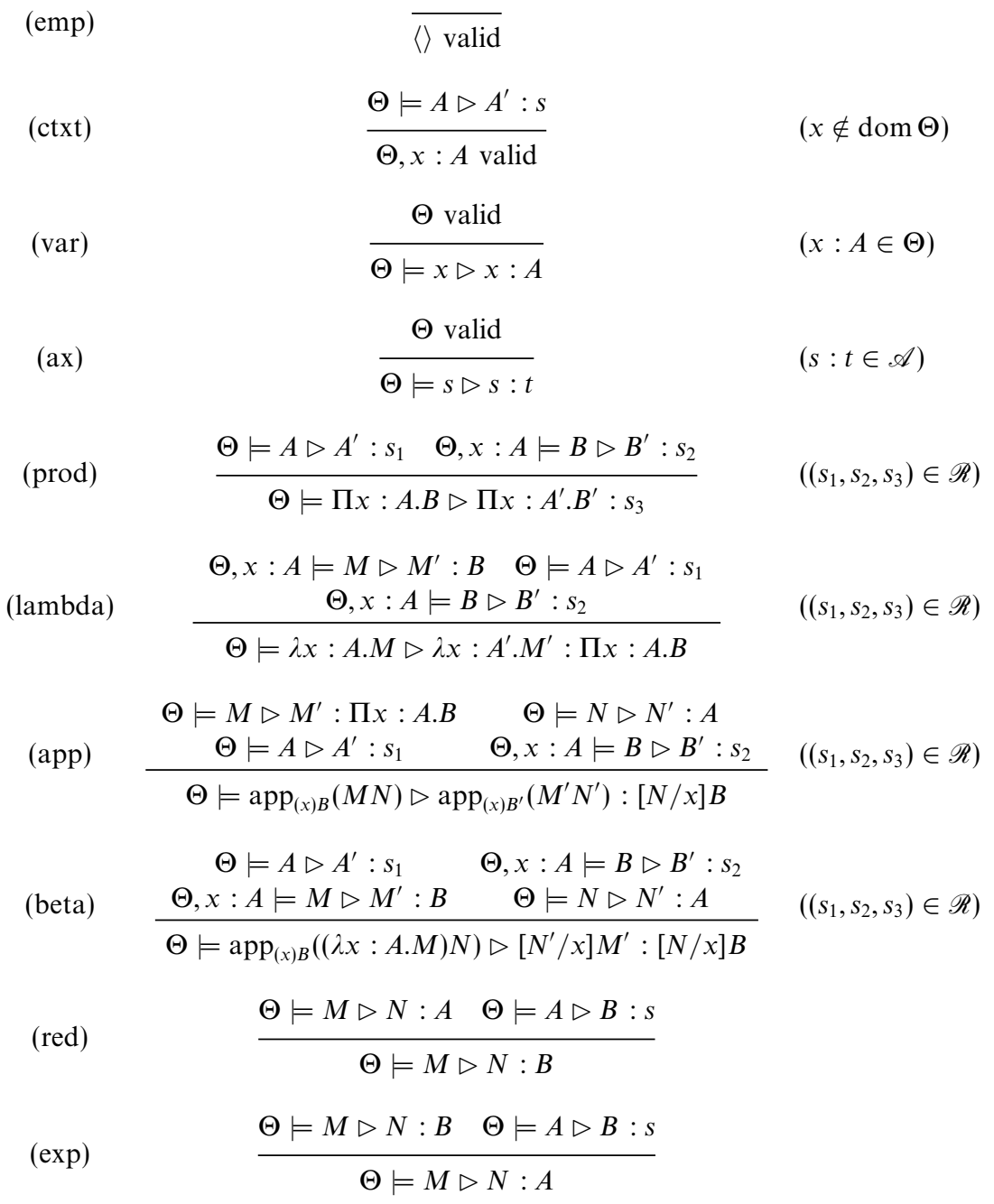

Fig. 4. Typed parallel one-step reduction.

\section{Proof}

We prove:

1. If $\Theta$ valid then

$$
\begin{gathered}
(\forall s: t \in \mathscr{A})|\Theta| \vdash^{e} s: t \\
(\forall x: A \in \Theta)|\Theta| \vdash^{e} x:|A|
\end{gathered}
$$

2. If $\Theta \mid=M \triangleright N: A$, then $|\Theta| \vdash^{e}|M|=|N|:|A|$.

simultaneously by induction on the derivation of the premise. All cases are straightforward. 
We prove several other metatheoretic results about typed parallel one-step reduction in the Appendix C.

\subsection{The Church-Rosser theorem}

We need the following result before we are able to prove the Church-Rosser Theorem for TPOSR. From now on, we shall always be assuming that $\mathbf{S}$ is a functional specification.

Lemma 3.6 (Uniqueness of Types)

Suppose $\mathbf{S}$ is a functional specification. If $\Theta \models M \triangleright$ ? : $A$ and $\Theta \models M \triangleright ?: B$, then either $A \equiv B$ or there is a sort $s$ such that $\Gamma \models A \simeq B: s$.

Proof

The proof is by induction on $M$, using Generation (Lemma C.15).

The case where $M$ is a $\lambda$-term uses Uniqueness of Sorts (Corollary C.1.4) and the Functionality of $\Pi$ (Lemma C.17).

In the case $M \equiv \operatorname{app}_{(x) C}(N P)$, Generation shows that $A$ and $B$ are each convertible with $[P / x] C$ within their respective sorts; Uniqueness of Sorts then gives the result we need.

We are now able to prove the diamond property for typed parallel one-step reduction in the following form.

Theorem 3.7 (Church-Rosser)

If $\Theta \models M \triangleright N: A$ and $\Theta \models M \triangleright P: B$, then there exists $Q$ such that

$$
\begin{array}{lll}
\Theta \models N \triangleright Q: A & \Theta \models P \triangleright Q: A \\
\Theta \models N \triangleright Q: B & \Theta \models P \triangleright Q: B
\end{array}
$$

Proof

The proof is by double induction on the two premises. The only difficult case is where one premise was derived using (app), and the other using (beta):

So we assume the last steps in the two derivations were

$$
\begin{aligned}
& \Theta \models A \triangleright A^{\prime}: s_{1} \quad \Theta, x: A=B \triangleright B^{\prime}: s_{2} \\
& \text { (beta) } \frac{\Theta, x: A=M \triangleright M^{\prime}: B \quad \Theta \models N \triangleright N^{\prime}: A}{\Theta \models \operatorname{app}_{(x) B}((\lambda x: A \cdot M) N) \triangleright\left[N^{\prime} / x\right] M^{\prime}:[N / x] B}\left(\left(s_{1}, s_{2}, s_{3}\right) \in \mathscr{R}\right)
\end{aligned}
$$

and

$$
\begin{aligned}
& \Theta \models \lambda x: A . M \triangleright P^{\prime \prime}: \Pi x: C . B \quad \Theta \models N \triangleright N^{\prime \prime}: C \\
& \text { (app) } \frac{\Theta \models C \triangleright C^{\prime \prime}: t_{1} \quad \Theta, x: C \models B \triangleright B^{\prime \prime}: t_{2}}{\Theta \models \operatorname{app}_{(x) B}((\lambda x: A . M) N) \triangleright \operatorname{app}_{(x) B^{\prime \prime}}\left(P^{\prime \prime} N^{\prime \prime}\right):[N / x] B}\left(\left(t_{1}, t_{2}, t_{3}\right) \in \mathscr{R}\right)
\end{aligned}
$$

Now, Uniqueness of Types gives either $A \equiv C$ or $\Theta=A \simeq C: t$ for some sort $t$; in either case, Uniqueness of Sorts gives

$$
s_{1} \equiv t_{1}
$$


Further, by Context Conversion,

$$
\Theta, x: A \mid=B \triangleright B^{\prime \prime}: t_{2}, \quad \Theta, x: C=B \triangleright B^{\prime}: s_{2},
$$

and so Uniqueness of Sorts gives

$$
s_{2} \equiv t_{2}
$$

(Functionality therefore gives $s_{3} \equiv t_{3}$, but we shall not need this fact.)

Applying Generation to the premise $\Theta=\lambda x: A . M \triangleright P^{\prime \prime}: \Pi x: C . B$, there exist $A^{\prime \prime}, M^{\prime \prime}, D, D^{\prime \prime}$ such that the given derivation of that premise has subderivations of

$$
\begin{aligned}
\Theta & =A \triangleright A^{\prime \prime}: s_{1} \\
\Theta, x: A & =M \triangleright M^{\prime \prime}: D \\
\Theta, x: A & =D \triangleright D^{\prime \prime}: s_{2}
\end{aligned}
$$

Further, $P^{\prime \prime} \equiv \lambda x: A^{\prime \prime} . M^{\prime \prime}$, and $\Theta \models \Pi x: C . B \simeq \Pi x: A . D: s_{3}$. (Again, Uniqueness of Sorts shows that it must be the same rule $\left(s_{1}, s_{2}, s_{3}\right)$ used here.)

By the induction hypothesis, we conclude that there exist labelled terms $A_{0}, B_{0}$, $M_{0}, N_{0}$ such that

$$
\begin{aligned}
\Theta & =A^{\prime} \triangleright A_{0}: s_{1} & \Theta & \models A^{\prime \prime} \triangleright A_{0}: s_{1} \\
\Theta, x: A & =B^{\prime} \triangleright B_{0}: s_{2} & \Theta, x: A & \models B^{\prime \prime} \triangleright B_{0}: s_{2} \\
\Theta, x: A & =M^{\prime} \triangleright M_{0}: B & \Theta, x: A & \models M^{\prime \prime} \triangleright M_{0}: B \\
\Theta & =N^{\prime} \triangleright N_{0}: A & \Theta & =N^{\prime \prime} \triangleright N_{0}: A
\end{aligned}
$$

(If (3) is the derivation of our first premise, then we have applied the induction hypothesis to its premises. If (4) is the derivation of our first premise, then we have applied the induction hypothesis both to its premises and to (6) and (7), which occur in the derivation of one of its premises.)

Substitution now gives

$$
\Theta \models\left[N^{\prime} / x\right] M^{\prime} \triangleright\left[N_{0} / x\right] M_{0}:\left[N^{\prime} / x\right] B .
$$

We can derive the judgements

$$
\begin{array}{ll}
\Theta, x: A^{\prime \prime} & =B^{\prime \prime} \triangleright B_{0}: s_{2} \\
\Theta, x: A^{\prime \prime} & =M^{\prime \prime} \triangleright M_{0}: B^{\prime \prime}
\end{array}
$$

and (beta) then gives

$$
\Theta \models \operatorname{app}_{(x) B^{\prime \prime}}\left(\left(\lambda x: A^{\prime \prime} . M^{\prime \prime}\right) N^{\prime \prime}\right) \triangleright\left[N_{0} / x\right] M_{0}:\left[N^{\prime \prime} / x\right] B^{\prime \prime} ;
$$

i.e.

$$
\Theta \models \operatorname{app}_{(x) B^{\prime \prime}}\left(P^{\prime \prime} N^{\prime \prime}\right) \triangleright\left[N_{0} / x\right] M_{0}:\left[N^{\prime \prime} / x\right] B^{\prime \prime} .
$$

It remains only to adjust the types of these two conclusions, using Reflexivity, Substitution and (exp).

Corollary 3.8

If $\Theta \models A \simeq B: s$, there exists $C$ such that $\Theta \models A \triangleright^{+} C: s$ and $\Theta \models B \triangleright^{+} C: s$. 
We have the following two very important corollaries of the Church-Rosser Theorem:

Corollary 3.9 (Injectivity of $\Pi$ )

If $\Theta \models \Pi x: A . B \simeq \Pi x: A^{\prime} \cdot B^{\prime}: s_{3}$, then there are sorts $s_{1}, s_{2}$ such that $\left(s_{1}, s_{2}, s_{3}\right) \in \mathscr{R}$ and

$$
\begin{aligned}
\Theta & =A \simeq A^{\prime}: s_{1} \\
\Theta, x: A & =B \simeq B^{\prime}: s_{2}
\end{aligned}
$$

Proof

By Church-Rosser, there are sequences of terms $X_{1}, \ldots, X_{m}$ and $Y_{1}, \ldots, Y_{n}$ such that

$$
\Theta \models \Pi x: A . B \triangleright X_{1} \triangleright X_{2} \triangleright \cdots \triangleright X_{m} \triangleright Z: s_{3}
$$

and

$$
\Theta \models \Pi x: A^{\prime} . B^{\prime} \triangleright Y_{1} \triangleright Y_{2} \triangleright \cdots \triangleright Y_{n} \triangleright Z: s_{3} .
$$

By Generation, each $X_{i}, Y_{j}$, and $Z$ itself is a $\Pi$-term; hence, by Generation and Uniqueness of Sorts, $A$ and $A^{\prime}$ both reduce to the domain of $C$, and $B$ and $B^{\prime}$ to its codomain.

Theorem 3.10 (Subject Reduction)

If $\Theta \models M \triangleright ?: A$ and $M \rightarrow{ }_{\beta}^{1} N$, then $\Theta \models M \triangleright N: A$.

Proof

Induction on the relation $M \rightarrow{ }_{\beta}^{1} N$, using Generation. The case $M \equiv N$ is handled by Left-Hand Reflexivity. All the remaining cases apart from the $\beta$-contraction case are straightforward.

The $\beta$-contraction case is handled as follows:

Suppose $M \equiv \operatorname{app}_{(x) C}((\lambda x: B . P) Q)$ and $N \equiv\left[Q^{\prime} / x\right] P^{\prime}$, where $P \rightarrow{ }_{\beta}^{1} P^{\prime}, Q \rightarrow{ }_{\beta}^{1} Q^{\prime}$. By Generation, there exists $D$ and a rule $\left(s_{1}, s_{2}, s_{3}\right) \in \mathscr{R}$ such that

$$
\begin{aligned}
\Theta & \models D \triangleright ?: s_{1} \\
\Theta & \models Q \triangleright ?: D \\
\Theta, x: D & \models C \triangleright ?: s_{2} \\
\Theta & \models A \simeq[Q / x] C: s_{2}
\end{aligned}
$$

and either

$$
\Theta \models \lambda x: B . P \triangleright ?: \Pi x: D . C
$$

or $D \equiv B$ and

$$
\Theta, x: B \models P \triangleright ?: C .
$$

In either case (using Generation in the first case), there exists $E$ such that

$$
\Theta, x: B \models P \triangleright ?: E, \quad \Theta \models \Pi x: B . E \simeq \Pi x: D . C: s_{3} .
$$


Therefore, by П-injectivity,

$$
\begin{aligned}
\Theta & =B \simeq D: s_{1} \\
\Theta, x: B & =E \simeq C: s_{2}
\end{aligned}
$$

The induction hypothesis gives us

$$
\begin{aligned}
\Theta & =Q \triangleright Q^{\prime}: D \\
\Theta, x: B & \models P \triangleright P^{\prime}: E \\
\therefore \Theta & \models \operatorname{app}_{(x) C}((\lambda x: B . P) Q) \triangleright\left[Q^{\prime} / x\right] P^{\prime}:[Q / x] C \quad \text { (beta) } \\
\therefore \Theta & =\operatorname{app}_{(x) C}((\lambda x: B . P) Q) \triangleright\left[Q^{\prime} / x\right] P^{\prime}: A \quad((\text { red }) \text { and (exp)) }
\end{aligned}
$$

\section{The translation from typed reduction to pure type systems}

Now that we are armed with the Church-Rosser Theorem, we can establish that the translation

$$
M \mapsto|M|
$$

is a sound and complete translation from TPOSR to PTSs, and also from TPOSR to PTSs with equality. Together, these two results will establish the correspondence between PTSs and PTSs with equality that we desire.

We first need to establish that two typable labelled terms that have identical translates are convertible, in the following sense:

\section{Lemma 4.1}

Suppose $\Theta \mid=M \triangleright$ ? : $A, \Theta \models N \triangleright$ ? : $B$, and $|M| \equiv|N|$. Then there exists $P$ such that

$$
\begin{array}{ll}
\Theta \models M \triangleright^{+} P: A & \Theta \models N \triangleright^{+} P: A \\
\Theta \models M \triangleright^{+} P: B & \Theta \models N \triangleright^{+} P: B
\end{array}
$$

Proof

The proof is by induction on $M$. We deal here with the most difficult case: $M \equiv \operatorname{app}_{(x) D}(P Q)$. Then $N \equiv \operatorname{app}_{(x) D^{\prime}}\left(P^{\prime} Q^{\prime}\right)$, where $|P| \equiv\left|P^{\prime}\right|$ and $|Q| \equiv\left|Q^{\prime}\right|$. By Generation, there exist labelled terms $C, C^{\prime}$ and rules $\left(s_{1}, s_{2}, s_{3}\right),\left(t_{1}, t_{2}, t_{3}\right) \in \mathscr{R}$ such that

$$
\begin{array}{rlrlll}
\Theta & =C \triangleright ?: s_{1} & \Theta & =C^{\prime} \triangleright ?: t_{1} \\
\Theta, x: C & =D \triangleright ?: s_{2} & \Theta, x: C^{\prime} & = & D^{\prime} \triangleright ?: t_{2} \\
\Theta & =Q \triangleright ?: C & \Theta & = & Q^{\prime} \triangleright ?: C^{\prime} \\
\Theta & =A \simeq[Q / x] D: s_{2} & \Theta & =B \simeq\left[Q^{\prime} / x\right] D^{\prime}: t_{2} \\
\Theta & =P \triangleright ?: \Pi x: D . C & \Theta & =P^{\prime} \rho ?: \Pi x: C^{\prime} \cdot D^{\prime}
\end{array}
$$

Therefore, by induction hypothesis, there exist $P^{*}, Q^{*}$ such that

$$
\begin{aligned}
& \Theta=P \triangleright^{+} P^{*}: \Pi x: C . D \\
& \Theta=P^{\prime} \triangleright^{+} P^{*}: \Pi x: C . D \\
& \Theta=P \triangleright^{+} P^{*}: \Pi x: C^{\prime} . D^{\prime} \\
& \Theta \models P^{\prime} \triangleright^{+} P^{*}: \Pi x: C^{\prime} . D^{\prime} \\
& \Theta=Q \triangleright^{+} Q^{*}: C \\
& \Theta \models Q^{\prime} \triangleright^{+} Q^{*}: C \\
& \Theta \models Q \triangleright^{+} Q^{*}: C^{\prime} \\
& \Theta=Q^{\prime} \triangleright^{+} Q^{*}: C^{\prime}
\end{aligned}
$$


Hence, by Uniqueness of Types, we have

$$
\Theta \models \Pi x: C . D \simeq \Pi x: C^{\prime} . D^{\prime}: s_{3}, \quad \Theta \models C \simeq C^{\prime}: s_{1}
$$

and also $s_{1} \equiv t_{1}, s_{2} \equiv t_{2}, s_{3} \equiv t_{3}$. By $\Pi$-injectivity,

$$
\Theta, x: C \models D \simeq D^{\prime}: s_{2} .
$$

Hence, by Church-Rosser, there exists $D^{*}$ such that

$$
\Theta, x: C=D \triangleright^{+} D^{*}: s_{2}, \quad \Theta, x: C \models D^{\prime} \triangleright^{+} D^{*}: s_{2} .
$$

Therefore,

$$
\begin{gathered}
\Theta=\operatorname{app}_{(x) D}(P Q) \triangleright^{+} \operatorname{app}_{(x) D^{*}}\left(P^{*} Q^{*}\right):[Q / x] D, \\
\Theta=\operatorname{app}_{(x) D^{\prime}}\left(P^{\prime} Q^{\prime}\right) \triangleright^{+} \operatorname{app}_{(x) D^{*}}\left(P^{*} Q^{*}\right):\left[Q^{\prime} / x\right] D^{\prime}
\end{gathered}
$$

As we also have $\Theta \models A \simeq[Q / x] D \simeq\left[Q^{\prime} / x\right] D^{\prime} \simeq B: s_{2}$, the desired conclusion follows.

\section{Corollary 4.2}

If $\Theta \models A \triangleright$ ? : $s, \Theta \models B \triangleright$ ? : $t$, and $|A| \equiv|B|$, then $s \equiv t$ and $\Theta \models A \simeq B: s$.

Corollary 4.3

If $\Theta \models M \triangleright N: A$, $\Phi$ valid, and $|\Theta| \equiv|\Phi|$, then $\Phi=M \triangleright N: A$.

Proof

By Corollary 4.2 and Context Conversion.

We are now ready to establish the correspondence between PTSs and TPOSR.

Theorem 4.4

If $\Gamma \vdash M: A$ then there exist $\Gamma^{+}, M^{+}, A^{+}$such that

$$
\begin{aligned}
\left|\Gamma^{+}\right| & \equiv \Gamma \\
\left|M^{+}\right| & \equiv M \\
\left|A^{+}\right| & \equiv A
\end{aligned}
$$

and

$$
\Gamma^{+} \models M^{+} \triangleright M^{+}: A^{+}
$$

Proof

Induction on $\Gamma \vdash M: A$. Most cases are straightforward now that we have Corollary 4.3. We deal here with the case

$$
\text { (conversion) } \frac{\Gamma \vdash M: A \quad \Gamma \vdash B: s}{\Gamma \vdash M: B}\left(A \simeq_{\beta} B\right)
$$


By the induction hypothesis, there exist $\Gamma^{+}, M^{+}, A^{+}, \Gamma_{2}^{+}, B^{+}$such that

$$
\begin{gathered}
\left|\Gamma^{+}\right| \equiv\left|\Gamma_{2}^{+}\right| \equiv \Gamma \\
\left|M^{+}\right| \equiv M \\
\left|A^{+}\right| \equiv A \\
\left|B^{+}\right| \equiv B \\
\Gamma^{+}=M^{+} \triangleright M^{+}: A^{+} \\
\Gamma_{2}^{+} \models B^{+} \triangleright B^{+}: s \\
\therefore \Gamma^{+}=B^{+} \triangleright B^{+}: s
\end{gathered}
$$

By Type Validity, either $A^{+}$is a sort or there is a sort $t$ such that

$$
\Gamma^{+} \models A^{+} \triangleright ?: t \text {. }
$$

Case 1: $A^{+}$is a sort. In this case, $A$ is a sort. Hence, $B \rightarrow_{\beta} A$, and so $B^{+} \rightarrow_{\beta} A^{+}$. Therefore, by Subject Reduction,

$$
\Gamma^{+}=B^{+} \triangleright^{+} A^{+}: s
$$

and so

$$
\Gamma^{+} \models M^{+} \triangleright M^{+}: B^{+}
$$

by $(\exp )$.

Case 2: $\Gamma^{+} \models A^{+} \triangleright$ ?: $t$ In this case, by Church-Rosser, there is an unlabelled term $C$ such that

$$
A \rightarrow_{\beta} C, \quad B \rightarrow_{\beta} C .
$$

By Lemma 3.3, there are labelled terms $C_{0}, C_{1}$ such that

$$
A^{+} \rightarrow_{\beta} C_{0}, \quad B^{+} \rightarrow_{\beta} C_{1}, \quad\left|C_{0}\right| \equiv\left|C_{1}\right| \equiv C .
$$

By Subject Reduction,

$$
\begin{aligned}
& \Gamma^{+} \models A^{+} \triangleright^{+} C_{0}: t \\
& \Gamma^{+} \models B^{+} \triangleright^{+} C_{1}: s
\end{aligned}
$$

Therefore, by Corollary 4.2,

$$
\Gamma^{+} \models C_{0} \simeq C_{1}: s
$$

(and also $s \equiv t$ ). Hence, using (red) and (exp) repeatedly,

$$
\Gamma^{+} \models M^{+} \triangleright M^{+}: B^{+} \text {. }
$$

Finally, we can put all our results together and prove our main theorem.

\section{Theorem 4.5}

1. If $\Gamma \vdash M: A$ then $\Gamma \vdash^{e} M: A$.

2. If $\Gamma \vdash M: A, \Gamma \vdash N: A$, and $M \simeq_{\beta} N$, then $\Gamma \vdash^{e} M=N: A$. 
Proof

1. By Theorem 4.4 , there exist $\Gamma^{+}, M^{+}, A^{+}$such that $\left|\Gamma^{+}\right| \equiv \Gamma,\left|M^{+}\right| \equiv M$, $\left|A^{+}\right| \equiv A$, and

$$
\begin{aligned}
& \Gamma^{+} \models M^{+} \triangleright M^{+}: A^{+} \\
& \therefore \Gamma \quad \vdash^{e} \quad M=M: A \quad \text { (Theorem 3.5) } \\
& \therefore \Gamma \quad \vdash^{e} \quad M: A \quad \text { (Equation Validity) }
\end{aligned}
$$

2. By Church-Rosser, there exists $P$ such that $M \rightarrow_{\beta} P$ and $N \rightarrow_{\beta} P$. By Theorem 4.4, there exist $\Gamma^{+}, M^{+}, A^{+}$such that $\left|\Gamma^{+}\right| \equiv \Gamma,\left|M^{+}\right| \equiv M,\left|A^{+}\right| \equiv A$, and

$$
\begin{aligned}
& \Gamma^{+} \models M^{+} \triangleright M^{+}: A^{+} \\
& \therefore \Gamma^{+} \models M^{+} \triangleright^{+} P^{+}: A^{+} \quad \text { (Subject Reduction) } \\
& \therefore \Gamma \quad \vdash^{e} \quad M=P: A \quad \text { (Theorem } 3.5 \text { and (trans)) }
\end{aligned}
$$

Similarly,

$$
\begin{aligned}
\Gamma \quad \vdash^{e} \quad N=P: A \\
\therefore \Gamma \quad \vdash^{e} \quad M=N: A \quad((\text { sym) and (trans)) }
\end{aligned}
$$

Theorem 4.6

For a functional PTS:

1. $\Gamma \vdash^{e} M: A$ iff $\Gamma \vdash M: A$.

2. $\Gamma \vdash^{e} M=N: A$ iff $\Gamma \vdash M: A, \Gamma \vdash N: A$ and $M={ }_{\beta} N$.

Proof

From Theorems 2.3 and 4.5 .

\section{Completing the theory}

To round off this long development, we make explicit the relationship between TPOSR and PTSs, and between TPOSR and PTSs with equality.

\section{Theorem 5.1}

1. If $\Theta \mid=M \triangleright N: A$, then $|\Theta| \vdash|M|:|A|,|\Theta| \vdash|N|:|A|$, and $|M| \rightarrow_{\beta}^{1}|N|$.

2. If $\Gamma \vdash M: A$ and $M \rightarrow{ }_{\beta}^{1} N$, then there exist $\Gamma^{+}, M^{+}, N^{+}, A^{+}$such that $\left|\Gamma^{+}\right| \equiv \Gamma,\left|M^{+}\right| \equiv M,\left|N^{+}\right| \equiv N,\left|A^{+}\right| \equiv A$, and

$$
\Gamma^{+} \models M^{+} \triangleright N^{+}: A^{+} \text {. }
$$

Proof

1. Follows from Lemmas 3.2, 3.5 and 2.3.

2. Follows from Theorems 4.5 and 3.10 .

\section{Theorem 5.2}

1. If $\Theta \mid=M \triangleright N: A$, then $|\Theta| \vdash^{e}|M|=|N|:|A|$ and $|M| \rightarrow_{\beta}^{1}|N|$.

2. If $\Gamma \vdash^{e} M: A$ and $M \rightarrow_{\beta}^{1} N$, then there exist $\Gamma^{+}, M^{+}, N^{+}, A^{+}$such that $\left|\Gamma^{+}\right| \equiv \Gamma,\left|M^{+}\right| \equiv M,\left|N^{+}\right| \equiv N,\left|A^{+}\right| \equiv A$, and

$$
\Gamma^{+} \models M^{+} \triangleright N^{+}: A^{+} \text {. }
$$


Proof

1. Follows from Lemmas 3.2 and 3.5.

2. Follows from Theorems 2.3, 4.5 and 3.10.

And so we are also able to complete the metatheory of PTSs with equality with the following results, which include the long promised Subject Reduction:

Corollary 5.3 (Subject Reduction)

If $\Gamma \vdash^{e} M: A$ and $M \rightarrow_{\beta} N$, then $\Gamma \vdash^{e} M=N: A$.

Proof

By our main result (Theorem 4.5), $\Gamma \vdash M: A$. By Subject Reduction for PTSs, $\Gamma \vdash N: A$. Therefore, by Theorem 4.5 again, $\Gamma \vdash^{e} M=N: A$.

Corollary 5.4 (Injectivity of $\Pi$ )

If $\Gamma \vdash^{e} \Pi x: A . B=\Pi x: A^{\prime} . B^{\prime}: s_{3}$, then there are sorts $s_{1}, s_{2}$ such that $\left(s_{1}, s_{2}, s_{3}\right) \in \mathscr{R}$ and

$$
\Gamma \vdash^{e} A=A^{\prime}: s_{1}, \quad \Gamma, x: A \vdash^{e} B=B^{\prime}: s_{2} .
$$

Proof

By Theorem 4.5, $\Gamma \vdash \Pi x: A . B: s_{3}$ and $\Gamma \vdash \Pi x: A^{\prime} \cdot B^{\prime}: s_{3}$. Therefore, by Generation for PTSs, there are sorts $s_{1}, s_{2}, t_{1}, t_{2}$ such that $\left(s_{1}, s_{2}, s_{3}\right),\left(t_{1}, t_{2}, s_{3}\right) \in \mathscr{R}$ and

$$
\begin{array}{rr}
\Gamma \vdash A: s_{1} & \Gamma \vdash A^{\prime}: t_{1} \\
\Gamma, x: A \vdash B: s_{2} & \Gamma, x: A^{\prime} \vdash B^{\prime}: t_{2}
\end{array}
$$

Using Uniqueness of Types and Context Conversion, it follows that $s_{1} \equiv t_{1}$ and $s_{2} \equiv t_{2}$. Therefore, by Theorem 4.5 ,

$$
\Gamma \vdash^{e} A=A^{\prime}: s_{1}, \quad \Gamma, x: A \vdash^{e} B=B^{\prime}: s_{2} .
$$

\section{Conclusion and future work}

We have proven the equivalence of a system with an external criterion for equality (the PTS) with one with a judgement form for equality (the PTS with equality) for all functional PTSs. It should be straightforward to extend the result to other type systems with the Uniqueness of Types property.

Such a technically complex proof should be checked mechanically. The author is working on a formalisation of this result in Coq, and has made his partial results available on his website.

The question naturally arises whether this approach could be applied to $\eta$ conversion, or to systems with a subtyping relation, such as ECC (Luo, 1994) or the Pure Type Systems with Universes, or $\gamma$ PTSs, studied by Ruiz (1999, 2000). These latter systems do not satisfy Uniqueness of Types; but, under appropriate hypotheses, every typable term has a unique principal type, and it may be that this is sufficient for the method to be applied.

It remains to be seen whether the relation of TPOSR can be applied in other areas of the theory of PTSs, or type systems in general. One idea might be to make 
some progress on the problem of expansion postponement (Poll, 1998) using the properties of the relation formed by leaving out the (exp) clause in the definition of TPOSR. Unfortunately, the properties of this relation prove difficult to establish. (See Gutiérrez \& Ruiz (2003) for the latest progress on expansion postponement.)

There is also an obvious superficial similarity between TPOSR and typed operational semantics (Goguen, 1999). It remains to be seen whether it would be useful to establish a more formal correspondence between the two.

The question of the equivalence of external and judgemental equality remains an open problem for an arbitrary PTS. It is a plausible enough conjecture: the rules for judgemental equality amount to little more than checking that a $\beta$-redex is well-typed whenever it is contracted; and, by Church-Rosser, for any two convertible well-typed terms, there is a reduction-expansion sequence between the two in which each redex is well-typed. However, it proves stubbornly difficult to establish in practise. It is quite possible that some pathological PTS will turn out to be a counterexample.

\section{A Reduction relations}

The relations

$\begin{array}{ll}\text { one-step beta reduction } & \rightarrow_{\beta} \\ \text { beta reduction } & \rightarrow_{\beta} \\ \text { beta conversion } & \simeq_{\beta} \\ \text { parallel one-step beta reduction } & \rightarrow_{\beta}^{1}\end{array}$

are defined on the set of unlabelled terms inductively by the following clauses:

One-step Beta Reduction

$$
\begin{array}{cc}
\overline{(\lambda x: A \cdot M) N \rightarrow \beta[N / x] M} \\
\overline{\Pi x: A . B \rightarrow_{\beta} A^{\prime} \Pi x: A^{\prime} \cdot B} & \frac{B \rightarrow_{\beta} B^{\prime}}{\Pi x: A \cdot B \rightarrow_{\beta} \Pi x: A \cdot B^{\prime}} \\
\frac{A \rightarrow_{\beta} A^{\prime}}{\lambda x: A . M \rightarrow_{\beta} \lambda x ; A^{\prime} \cdot M} & \frac{M \rightarrow_{\beta} M^{\prime}}{\lambda x: A \cdot M \rightarrow_{\beta} \lambda x: A \cdot M^{\prime}} \\
\frac{M \rightarrow_{\beta} M^{\prime}}{M N \rightarrow_{\beta} M^{\prime} N} & \frac{N \rightarrow_{\beta} N^{\prime}}{M N \rightarrow_{\beta} M N^{\prime}}
\end{array}
$$

Beta-Reduction

$$
\frac{M \rightarrow_{\beta} N}{M \rightarrow_{\beta} N} \quad \overline{M \rightarrow_{\beta} M} \quad \frac{M \rightarrow_{\beta} N \quad N \rightarrow_{\beta} P}{M \rightarrow_{\beta} P}
$$

Beta Conversion

$$
\frac{M \rightarrow_{\beta} N}{M \simeq_{\beta} N} \quad \overline{M \simeq_{\beta} M} \quad \frac{M \simeq_{\beta} N}{N \simeq_{\beta} M} \quad \frac{M \simeq_{\beta} N N \simeq_{\beta} P}{M \simeq_{\beta} P}
$$




\section{Parallel One-Step Beta Reduction}

$$
\begin{gathered}
\frac{A \rightarrow{ }_{\beta}^{1} A^{\prime} B \rightarrow{ }_{\beta}^{1} B^{\prime}}{M \rightarrow{ }_{\beta}^{1} M} \quad \frac{\frac{A}{\Pi x: A . B \rightarrow{ }_{\beta}^{1} \Pi x: A^{\prime} \cdot B^{\prime}}}{\lambda \rightarrow{ }_{\beta}^{1} A^{\prime} \quad M \rightarrow{ }_{\beta}^{1} M^{\prime}} \frac{M \rightarrow{ }_{\beta}^{1} M^{\prime} N \rightarrow{ }_{\beta}^{1} N^{\prime}}{M N \rightarrow{ }_{\beta}^{1} M^{\prime} N^{\prime}} \\
\frac{M x: A . M \rightarrow{ }_{\beta}^{1} \lambda x: A^{\prime} \cdot M^{\prime}}{M} \frac{{ }_{\beta}^{1} M^{\prime} \quad N \rightarrow{ }_{\beta}^{1} N^{\prime}}{(\lambda x: A \cdot M) N \rightarrow{ }_{\beta}^{1}\left[N^{\prime} / x\right] M^{\prime}}
\end{gathered}
$$

The relations

$$
\begin{array}{ll}
\text { one-step beta reduction } & \rightarrow_{\beta} \\
\text { beta reduction } & \rightarrow_{\beta} \\
\text { beta convertibility } & \simeq_{\beta} \\
\text { parallel one-step beta reduction } & \rightarrow_{\beta}^{1}
\end{array}
$$

are defined on the set of labelled terms as follows:

\section{One-step Beta Reduction}

$$
\begin{gathered}
\overline{\operatorname{app}_{(x) B}((\lambda x: A \cdot M) N) \rightarrow_{\beta}[N / x] M} \\
\frac{A \rightarrow_{\beta} A^{\prime}}{\Pi x: A . B \rightarrow_{\beta} \Pi x: A^{\prime} \cdot B} \quad \frac{B \rightarrow_{\beta} B^{\prime}}{\Pi x: A \cdot B \rightarrow_{\beta} \Pi x: A \cdot B^{\prime}} \\
\frac{A \rightarrow_{\beta} A^{\prime}}{\lambda x: A \cdot M \rightarrow_{\beta} \lambda x ; A^{\prime} \cdot M} \quad \frac{M \rightarrow_{\beta} M^{\prime}}{\lambda x: A \cdot M \rightarrow_{\beta} \lambda x: A \cdot M^{\prime}} \\
\frac{\operatorname{app}_{(x) A}(M N) \rightarrow_{\beta} \operatorname{app}_{(x) A}\left(M^{\prime} N\right)}{\operatorname{app}_{(x) A}(M N) \rightarrow_{\beta} \operatorname{app}_{(x) A}\left(M N^{\prime}\right)} \\
\operatorname{app}_{(x) A}(M N) \rightarrow_{\beta} \operatorname{app}_{(x) A^{\prime}}(M N)
\end{gathered}
$$

Beta-Reduction

$$
\frac{M \rightarrow_{\beta} N}{M \rightarrow_{\beta} N} \quad \overline{M \rightarrow_{\beta} M} \quad \frac{M \rightarrow_{\beta} N \quad N \rightarrow_{\beta} P}{M \rightarrow_{\beta} P}
$$

Beta Conversion

$$
\frac{M \rightarrow_{\beta} N}{M \simeq_{\beta} N} \quad \overline{M \simeq_{\beta} M} \quad \frac{M \simeq_{\beta} N}{N \simeq_{\beta} M} \quad \frac{M \simeq_{\beta} N N \simeq_{\beta} P}{M \simeq_{\beta} P}
$$


Parallel One-Step Beta Reduction

$$
\begin{aligned}
& \overline{M \rightarrow{ }_{\beta}^{1} M} \quad \frac{A \rightarrow{ }_{\beta}^{1} A^{\prime} \quad B \rightarrow{ }_{\beta}^{1} B^{\prime}}{\Pi x: A \cdot B \rightarrow_{\beta}^{1} \Pi x: A^{\prime} \cdot B^{\prime}} \\
& \frac{A \rightarrow{ }_{\beta}^{1} A^{\prime} M \rightarrow{ }_{\beta}^{1} M^{\prime}}{\lambda x: A . M \rightarrow{ }_{\beta}^{1} \lambda x: A^{\prime} \cdot M^{\prime}} \quad \frac{A \rightarrow{ }_{\beta}^{1} A^{\prime} \quad M \rightarrow{ }_{\beta}^{1} M^{\prime} \quad N \rightarrow{ }_{\beta}^{1} N^{\prime}}{\operatorname{app}_{(x) A}(M N) \rightarrow_{\beta}^{1} \operatorname{app}_{(x) A^{\prime}}\left(M^{\prime} N^{\prime}\right)} \\
& \frac{M \rightarrow{ }_{\beta}^{1} M^{\prime} \quad N \rightarrow{ }_{\beta}^{1} N^{\prime}}{\operatorname{app}_{(x) B}((\lambda x: A \cdot M) N) \rightarrow{ }_{\beta}^{1}\left[N^{\prime} / x\right] M^{\prime}}
\end{aligned}
$$

\section{B Metatheory of pure type systems with equality}

The following are the results about pure type systems with equality used in this paper. They can be proven in the following order; each proof is by induction on the first-mentioned premise, making use of the previous results.

\section{Lemma B.1 (Free Variables and Contexts)}

1. If $\Gamma \vdash^{e} M: A$, then $\Gamma$ is consistent and $\mathrm{FV}(M) \cup \mathrm{FV}(A) \subseteq \operatorname{dom} \Gamma$.

2. If $\Gamma \vdash^{e} M=N: A$, then $\Gamma$ is consistent and FV $(M) \cup F V(N) \cup F V(A) \subseteq \operatorname{dom} \Gamma$.

Lemma B.2 (Context Conversion, Preliminary Form)

If $\Gamma, x: A, \Delta \vdash^{e} J, \Gamma \vdash^{e} A=B: s$ and $\Gamma \vdash^{e} B: s$, then $\Gamma, x: B, \Delta \vdash^{e} J$.

\section{Lemma B.3 (Substitution)}

If $\Gamma, x: A, \Delta \vdash^{e} J$ and $\Gamma \vdash^{e} M: A$, then $\Gamma,[M / x] \Delta \vdash^{e}[M / x] J$.

Lemma B.4 (Functionality, Preliminary Form)

If $\Gamma, x: A, \Delta \vdash^{e} M: B, \Gamma \vdash^{e} N=P: A$, and $\Gamma \vdash^{e} N: A$, then $\Gamma,[N / x] \Delta \vdash^{e}$ $[N / x] M=[P / x] M:[N / x] B$.

\section{Definition B.5}

We shall write " $\Gamma \vdash^{e} X \simeq Y$ " for "Either $X \equiv Y$, or there exists a sequence of terms $X_{1}, \ldots, X_{n}$ and a sequence of terms $A_{0}, A_{1}, \ldots, A_{n}$ such that

$$
\Gamma \vdash^{e} X=X_{1}: A_{0}, \Gamma \vdash^{e} X_{1}=X_{2}: A_{1}, \ldots, \Gamma \vdash^{e} X_{n}=Y: A_{n} . "
$$

\section{Lemma B.6 (Generation)}

1. If $\Gamma \vdash^{e} s: A$, then there is a sort $t$ such that $s: t \in \mathscr{A}$, and $\Gamma \vdash^{e} A \simeq t$.

2. If $\Gamma \vdash^{e} x: A$, then $x: B \in \Gamma$ for some $B$, and $\Gamma \vdash^{e} A \simeq B$

3. If $\Gamma \vdash^{e} \Pi x: A . B: C$, then there is a rule $\left(s_{1}, s_{2}, s_{3}\right) \in \mathscr{R}$ such that $\Gamma \vdash^{e} A: s_{1}$, $\Gamma, x: A \vdash^{e} B: s_{2}$, and $\Gamma \vdash^{e} C \simeq s_{3}$.

4. If $\Gamma \vdash^{e} \lambda x: A . M: B$, then there is a rule $\left(s_{1}, s_{2}, s_{3}\right) \in \mathscr{R}$ and term $C$ such that $\Gamma \vdash^{e} A: s_{1}, \Gamma, x: A \vdash^{e} C: s_{2}, \Gamma, x: A \vdash^{e} M: C$, and $\Gamma \vdash^{e} B \simeq \Pi x: A . C$.

5. If $\Gamma \vdash^{e} M N: C$, then there are terms $A$ and $B$ such that $\Gamma \vdash^{e} M: \Pi x: A . B$, $\Gamma \vdash^{e} N: A$, and $\Gamma \vdash^{e}[N / x] B \simeq C$. 


\section{Lemma B.7 (Type Validity and Equation Validity)}

1. If $\Gamma \vdash^{e} M: A$, then either $A$ is a sort, or there is a sort $s$ such that $\Gamma \vdash^{e} A: s$.

2. If $\Gamma \vdash^{e} M=N: A$ then $\Gamma \vdash^{e} M: A, \Gamma \vdash^{e} N: A$, and either $A$ is a sort, or there is a sort $s$ such that $\Gamma \vdash^{e} A: s$.

Now that we have Equation Validity, we can remove one premise from two previous lemmas:

Corollary B.8 (Context Conversion)

If $\Gamma, x: A, \Delta \vdash^{e} J$ and $\Gamma \vdash^{e} A=B: s$, then $\Gamma, x: B, \Delta \vdash^{e} J$.

Corollary B.9 (Functionality)

If $\Gamma, x: A, \Delta \vdash^{e} M: B$ and $\Gamma \vdash^{e} N=P: A$, then $\Gamma,[N / x] \Delta \vdash^{e}[N / x] M=[P / x] M$ : $[N / x] B$.

\section{Metatheory of typed parallel one-step reduction}

The following are the results about the relation of TPOSR used in the paper. In most cases, the proof is a straightforward induction on the derivation of the first premise given, making use of the previously proven lemmas.

Firstly, we can take advantage of the correspondence between TPOSR and PTSs, and the Uniqueness of Types result for PTSs, to prove a Uniqueness of Sorts lemma for typed parallel one-step reduction:

\section{Corollary C.1}

1. If $\Theta \mid=M \triangleright N: A$ then $|\Theta| \vdash|M|:|A|$ and $|\Theta| \vdash|N|:|A|$.

2. If $\Theta \mid=A \simeq B: s$ then $|\Theta| \vdash|A|: s$ and $|\Theta| \vdash|B|: s$.

3. Suppose $\mathbf{S}$ is a functional specification. If $\Theta \models A \triangleright B: s$ and $\Theta \models A \triangleright C: t$, then $s \equiv t$.

4. Suppose $\mathbf{S}$ is a functional specification. If $\Theta=A \simeq B: s$ and $\Theta \models A \simeq C: t$, then $s \equiv t$.

\section{Proof}

Part 1 is a consequence of Theorems 3.5 and 2.3; part 2 follows easily by induction on the premise. Parts 3 and 4 follow from parts 1 and 2 respectively, and the Uniqueness of Types result proven in (Barendregt, 1992).

It is clear that typed reduction entails untyped reduction:

Lemma C.2

If $\Theta \models X \triangleright Y: Z$ then $X \rightarrow_{\beta} Y$.

Corollary C.3

If $\Theta \models X \simeq Y: s$ then $X \simeq_{\beta} Y$.

Lemma C.4 (Context Validity)

If $\Theta, \Phi \models J$, then $\Theta$ valid.

Lemma C.5 (Free Variables)

If $x_{1}: X_{1}, \ldots, x_{n}: X_{n} \models J$, then $F V\left(X_{i}\right) \subseteq\left\{x_{1}, \ldots, x_{i-1}\right\}$ and $F V(J) \subseteq\left\{x_{1}, \ldots, x_{n}\right\}$. 
Lemma C.6 (Weakening)

If $\Theta \models X \triangleright Y: Z, \Theta \subseteq \Phi$, and $\Theta$ valid, then $\Theta \models X \triangleright Y: Z$.

Lemma C.7 (Context Reduction, Preliminary Form)

If $\Theta, x: X, \Phi=J, \Theta \models X \triangleright X^{\prime}: s$, and $\Theta \models X^{\prime} \triangleright X^{\prime}: s$, then $\Theta, x: X^{\prime}, \Phi \models J$.

Lemma C.8 (Left-Hand Reflexivity)

If $\Theta \models X \triangleright Y: Z$ then $\Theta=X \triangleright X: Z$.

Lemma C.9 (Substitution)

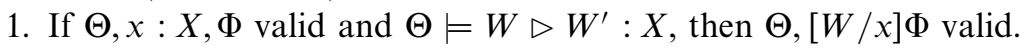

2. If $\Theta, x: X, \Phi \models Y \triangleright Y^{\prime}: Z$ and $\Theta \models W \triangleright W^{\prime}: X$, then $\Theta,[W / x] \Phi=$ $[W / x] Y \triangleright\left[W^{\prime} / x\right] Y^{\prime}:[W / x] Z$.

Lemma C.10 (Right-Hand Reflexivity)

If $\Theta \models X \triangleright Y: Z$ then $\Theta \models Y \triangleright Y: Z$.

Corollary C.11

If $\Theta \models X \simeq Y: s$, then $\Theta \models X \triangleright X: s$ and $\Theta \models Y \triangleright Y: s$.

Right-Hand Reflexivity allows us to remove one of the premises in Context Reduction:

Corollary C.12 (Context Reduction)

If $\Theta, x: X, \Phi \models J$ and $\Theta \models X \triangleright X^{\prime}: s$, then $\Theta, x: X^{\prime}, \Phi \models J$.

Lemma C.13 (Context Expansion)

If $\Theta, x: X^{\prime}, \Phi \models J$ and $\Theta \models X \triangleright X^{\prime}: s$, then $\Theta, x: X, \Phi \models J$.

Corollary C.14 (Context Conversion)

If $\Theta, x: X, \Psi \models J$ and $\Theta \models X \simeq Y: s$, then $\Theta, x: Y, \Psi \models J$.

Lemma C.15 (Generation)

Suppose $\mathbf{S}$ is a functional specification.

1. If $\Theta=s \triangleright X: Y$, then $X \equiv s$, and there exists $t \in \mathscr{S}$ such that $s: t \in \mathscr{A}$, and either $Y \equiv t$ or $\Theta \models Y \simeq t: t^{\prime}$ for some sort $t^{\prime}$.

2. If $\Theta \mid=x \triangleright X: Y$, then $X \equiv x$, and there exist $Z$, s such that $x: Z \in \Theta$ and $\Theta \models Y \simeq Z: s$.

3. Any derivation of $\Theta=\Pi x: W . X \triangleright Y: Z$ has subderivations of

$$
\begin{aligned}
\Theta & =W \triangleright W^{\prime}: s_{1} \\
\Theta, x: W & =X \triangleright X^{\prime}: s_{2}
\end{aligned}
$$

for some labelled terms $W^{\prime}, X^{\prime}$ and rule $\left(s_{1}, s_{2}, s_{3}\right) \in \mathscr{R}$; further, $Y \equiv \Pi x$ : $W^{\prime} \cdot X^{\prime}$, and either $Z \equiv s_{3}$, or $\Theta \models Z \simeq s_{3}: t$ for some sort $t$.

4. Any derivation of $\Theta=\lambda x: W . X \triangleright Y: Z$ has subderivations of

$$
\begin{aligned}
\Theta & =W \triangleright W^{\prime}: s_{1} \\
\Theta, x: W & =V \triangleright V^{\prime}: s_{2} \\
\Theta, x: W & =X \triangleright X^{\prime}: V
\end{aligned}
$$

for some labelled terms $W^{\prime}, X^{\prime}, V, V^{\prime}$ and rule $\left(s_{1}, s_{2}, s_{3}\right) \in \mathscr{R}$; further, $Y \equiv \lambda x: W^{\prime} \cdot X^{\prime}$, and $\Theta=Z \simeq \Pi x: W . V: s_{3}$. 
5. Any derivation $\mathscr{D}$ of $\Theta \models \operatorname{app}_{(x) V}(W X) \triangleright Y: Z$ has subderivations of

$$
\begin{aligned}
\Theta & \models U \triangleright U^{\prime}: s_{1} \\
\Theta, x: U & \models V \triangleright V^{\prime}: s_{2} \\
\Theta & \models X \triangleright X^{\prime}: U
\end{aligned}
$$

for some labelled terms $X^{\prime}, U, U^{\prime}, V^{\prime}$ and rule $\left(s_{1}, s_{2}, s_{3}\right) \in \mathscr{R}$. Furthermore,

$$
\Theta \models Z \simeq[X / x] V: s_{2}
$$

and either

(a) there exists $W^{\prime}$ such that $\mathscr{D}$ has a subderivation of

$$
\Theta \models W \triangleright W^{\prime}: \Pi x: U . V
$$

and $Y \equiv \operatorname{app}_{(x) V^{\prime}}\left(W^{\prime} X^{\prime}\right)$; or

(b) $W \equiv \lambda x: U . T$, and there exists $T^{\prime}$ such that $\mathscr{D}$ has a subderivation of

$$
\Theta, x: U \models T \triangleright T^{\prime}: V
$$

and $Y \equiv\left[X^{\prime} / x\right] T^{\prime}$.

\section{Proof}

Straightforward induction on the premise in each case, using the fact that a type can be in only one sort.

Without the assumption of functionality, we would need to replace each conclusion of the form $\Theta \mid=X \simeq Y: s$ with "There exist a sequence of labelled terms $Z_{1}, \ldots, Z_{n}$, and a sequence of sorts $s_{1}, \ldots, s_{n+1}$, such that

$$
\Theta \models X \simeq Z_{1}: s_{1}, \Theta \models Z_{1} \simeq Z_{2}: s_{2}, \cdots, \Theta \mid=Z_{n} \simeq Y: s_{n+1} . "
$$

Corollary C.16 (Type Validity)

If $\Theta=X \triangleright Y: Z$, then either $Z$ is a sort, or there is a sort $s$ and labelled term $Z^{\prime}$ such that $\Theta=Z \triangleright Z^{\prime}$ : s.

Proof

Inspection of each case of Generation.

Lemma C.17 (Functionality of $\Pi$ )

If $\left(s_{1}, s_{2}, s_{3}\right) \in \mathscr{R}, \Theta \models X \simeq X^{\prime}: s_{1}$, and $\Theta, x: X \models Y \simeq Y^{\prime}: s_{2}$, then $\Theta \models \Pi x:$ $X . Y \simeq \Pi x: X^{\prime} . Y^{\prime}: s_{3}$.

\section{Proof}

We prove the two statements:

1. If $\Theta \models X \triangleright X: s_{1}$ and $\Theta, x: X \models Y \simeq Y^{\prime}: s_{2}$, then $\Theta \models \Pi x: X . Y \simeq \Pi x:$ $X . Y^{\prime}: s_{3}$.

2. If $\Theta \models X \simeq X^{\prime}: s_{1}$ and $\Theta, x: X \models Y \triangleright Y: s_{2}$, then $\Theta \models \Pi x: X . Y \simeq \Pi x:$ $X^{\prime} . Y: s_{3}$.

These, together with Reflexivity, suffice to prove the lemma. The proofs are by induction on $\Theta, x: X \mid=Y \simeq Y^{\prime}: s_{2}$ in the first case, and $\Theta=X \simeq X^{\prime}: s_{1}$ in the second. 


\section{References}

Barendregt, H. (1992) Lambda calculi with types. In: Abramsky, S., Gabbay, D. M. and Maibaum, T. S. E. (eds.), Handbook of Logic in Computer Science, vol. II. Oxford University Press.

Coquand, T. (1991) An algorithm for testing conversion in type theory. In: Huet, G. and Plotkin, G. (eds.), Logical Frameworks, pp. 255-279. Cambridge University Press.

Coquand, T. and Huet, G. (1988) The calculus of constructions. Infor. \& Computation, 76, 95-120.

Geuvers, H. (1993) Logics and type systems. PhD thesis, Katholieke Universiteit Nijmegen.

Goguen, H. (1994) A typed operational semantics for type theory. PhD thesis, Edinburgh University.

Goguen, H. (1999) Soundness of typed operational semantics for the logical framework. In: Girard, Je.-Y. (ed)., Typed Lambda Calculi and Applications, 4th International Conference TLCA'99, pp. 177-197. Lecture Notes in Computer Science, vol. 1581. Springer.

Gutiérrez, F. and Ruiz, B. C. (2003) Expansion postponement via cut elimination in sequence calculi for pure type systems. Thirtieth International Colloquium on Automata, Languages and Programming (ICALP'03). Lecture Notes in Computer Science, vol. 2719.

Harper, R., Honsell, F. and Plotkin, G. (1993) A framework for defining logics. J. ACM, 40(1), 143-184. (A preliminary version appeared in the Proceedings of the Symposium on Logic in Computer Science, pages 194-204, June 1987.)

Luo, Z. (1994) Computation and Reasoning: A type theory for computer science. International Series of Monographs on Computer Science, no. 11. Oxford University Press.

Luo, Z. (2003) $\mathrm{PAL}^{+}$: a lambda-free logical framework. J. Funct. Program. 13(2), 317-338.

Nordström, B., Petersson, K. and Smith, J. (1990) Programming in Martin-Löf's Type Theory: an introduction. Oxford University Press.

Poll, E. (1998) Expansion postponement for normalising pure type systems. J. Funct. Program. 8(1), 89-96.

Ruiz, B. C. (1999) Condensing lemmas in pure type systems with universes. In: Haeberer, A. M. (ed.), 7th International Conference on Algebraic Methodology and Software Technology (AMAST'98) Proceedings, pp. 422-437. Lecture Notes in Computer Science, vol. 1548. Springer-Verlag.

Ruiz, B. C. (2000) The expansion postponement problem in pure type systems with universes. In: 9th International Workshop on Functional and Logic Programming (WFLP'2000), pp. 210-224. Benicassim, Spain. Publication 2000/2039.

Streicher, T. (1991) Semantics of type theory: Correctness, completeness and independence results. Progress in Theoretical Computer Science. Boston: Birkhäuser.

van Benthem Jutting, L. S. (1993) Typing in pure type systems. Infor. \& Computation, 105(1), 30-41. 